\title{
PROCESOS ARTÍSTICOS EN LABORATORIOS. GÉNESIS Y PERSPECTIVAS
}

\author{
Artistic process in laboratories. Genesis and prospects
}

\author{
Reinaldo Villar Alé*
}

\section{RESUMEN}

Actualmente se observa un debate extenso sobre el fomento y el uso de la tecnología digital, del ciberespacio y de los medios de comunicación en la producción de arte. Imbricado con esa temática se apuesta, en el plano artístico, productivo y académico, por un espacio de trabajo siguiendo el modelo denominado Laboratorio (Lab), para hacer la diferencia con los términos de estudio o taller. El Laboratorio se presenta en una vasta combinatoria de nombres (Media Lab, Living Lab, City Lab, Hack Lab, etc) al mismo tiempo que se ha difundido una idea sobre la génesis de ellos asociada a una óptica angloeurocentrista de corte formalista y aspiración progresista. Sin embargo, existen también otros discursos y lugares en los que el concepto de laboratorio experimental jugó un rol activador de opciones antiestéticas y político social evidentes. El debate ha ignorado esas líneas genealógicas producto de un momento histórico crucial, cuyos integrantes produjeron una rica y profunda reflexión, conscientes en el fondo de la contradicción entre el uso de nuevas tecnologías en arte y estructuras socioeconómicas y culturales conservadoras. Finalmente, se ahonda en la pertinencia de dos conceptos asociados a los Laboratorios, el de colaboración y el de integración de áreas del conocimiento.

Palabras clave: Espacios arte actual, laboratorios experimentales, fundamentos e ideología.

\footnotetext{
* Doctor de la Universidad de Valladolid, España. Trabaja en Planificación y Desarrollo para el Gobierno Regional del Maule. Talca, Chile. También realiza investigación independiente. Correo electrónico: rvillar@goremaule.cl
}

Artículo recibido el 9 de diciembre de 2013. Aceptado el 20 de enero de 2015. 


\begin{abstract}
Nowadays we observe a wide debate about encouraging and using digital technology, cyberspace and media in art production. Overlapped with this issue, in the artistic, productive and academic fields, the bet has been placed on a workspace that follows the model called Laboratory (Lab), for making the difference with the term "studio". The Laboratory is presented in a vast names combination (Media Lab, Living Lab, City Lab, Hack Lab, etc) and at the same time it has been spread an idea about the genesis of them asociated with an anglo-euro-centrist point of view, formalist and with a progressive aspiration. However, there are other speeches and latitudes where the conceptof experimental laboratory played a rol of activating evident unaesthetic and sociopolitical options. The debate has ignored those genealogical lines because of a crucial historical moment, whose members produced a rich and deep reflection, aware of the contradiction between the use of new technologies in art and conservative socio-economic and cultural structures. Finally, it has been analyzed in depth the apropriateness of two concepts asociated to Labs: colaboration and knowledge areas integration.
\end{abstract}

Keywords: New art spaces, current art spaces, experimental laboratories, fundaments and ideology.

\title{
INTRODUCCIÓN
}

El enfoque que se esboza busca aclarar la idea de producción de arte a través del Laboratorio, señalando para ello dos recorridos genealógicos que difieren en sus objetivos últimos y planteamientos ideológicos.

Por un lado, tomaremos los antecedentes tempranos, que proponen el Constructivismo-Productivista de entre 1910-1927, y otro proveniente de una universidad estadounidense a mediados de los años ochenta, mucho más presente en los medios comunicacionales en la actualidad occidental. Dos opciones que parecen tener similitudes, pero muy diferentes en su discurso, su relación con la sociedad y su pertinencia con la producción de objetos de diseńo y arte. Consideramos el término producción de arte por aquel que representa los métodos de producción, el ensayo, el tratamiento de imágenes, el desarrollo de conceptos, la experimentación y las pruebas (Borgdorff, 2006). Por otro lado, recurrimos a una reflexión de Benjamin (1990), al situarnos en una distinción clave, de dos caras, entre los métodos de producción de arte, en el contexto del arte de los años treinta. La primera sería la que atañe a las producciones de arte preocupados por lo social, reflejado en una concentración por una temática política-popular. Entre los segundos, estarían aquellas producciones que priorizan la técnica, la función y el aparato de producción. Esencialmente Benjamin manifiesta que se han descuidado 
las condiciones de lo segundo, pero que resulta ser un elemento imprescindible para posicionarse en dichas condiciones y conseguir el gesto político transformador.

Actualmente, en este plano de la producción y los lugares donde ocurre, existe una serie de factores y discursos que se unen para presentar el fenómeno de los Laboratorios y su metodología como producción innovadora con nuevas herramientas tecnológicas, interdisciplinar e información extendida. Se apela a que el término Laboratorio ya no sería exclusivo de los centros científicos, o de la universidad, presentándose bajo una enorme variedad y densidad de proyectos y textos no exentos de imprecisiones. A diferencia del icónico del estudio de artista, actualmente está en boga el sufijo $L a b$ y es utilizado, por algunos grupos, poniéndolo como un hito cardinal dentro de la esfera de innovación. El cual tendría sus raíces en: A) los laboratorios científicos, B) el laboratorio industrial, C) el laboratorio de diseño y D) el laboratorio tecnológico digital (Sangüesa, 2013). Al mismo tiempo que adscritos al "usuario avanzado" y a la "innovación abierta" (Serra, 2013:285). Sin embargo, rara vez se señalan antecedentes en profundidad sobre otras experiencias similares. Por ejemplo, las tesis propuestas en la exposición colectiva de 1910 de la Unión de la Juventud en San Petersburgo, quienes con sus producciones experimentales sientan las bases de una imbricación entre una estética, un proceso de producción y las relaciones sociales (Lodder, 1988). En el mismo sentido es la propuesta del Laboratorio Experimental de Construcciones Cinéticas del Proletkult moscovita (Raunig, 2009). En dicho laboratorio se trató de activar los nuevos medios de comunicación en espacios colectivos experimentales para organizar una producción artística diferente a la elitista, concordando una correspondencia de intercambio transversal.

Entonces por qué en la actualidad se debate el tema de los laboratorios considerando antecedentes parcializados ¿Cuál es el afán de acceder a un estatus de innovación de perfil científico y poner la colaboración como identidad de estos espacios? ¿Por qué se asocian a lo híbrido, conectado con el público, renovado y ciudadano? Se agrega a ello que la intervención e invención del New Media Art con los $L a b$, proyectan una ambigua definición de lo nuevo. ¿Cómo delimitar estos $L a b$, cómo se fundamenta y dónde pone el objetivo? ¿Cuánto hay de interacción con el arte, la ciencia, la tecnología o la mercancía en ellos? ¿Cuáles son ejemplos de Labs en los contextos del arte actual y su relevancia como espacio de participación y emancipación? ¿Cuál es el trasfondo del concepto de laboratorio?

En un primer instante podemos señalar que el término $L a b$ se ajusta, básicamente, al uso de Tecnologías de la Información y Comunicación (TIC) en un espacio-taller determinado, con una metodología de trabajo grupal e interdisciplinar, con actividades y producciones que pueden situarse entre el activismo social y la producción de objetos.

Asimismo se puede agregar que existen referencias del afán de fusión de arte y máquinas desde fines del siglo XIX y más significativo para un enfoque 
actualizado, cuando se fusiona el uso de herramientas como la radio, la fotografía, el cine, la telefonía, etc, y los cuestionamientos artísticos e investigaciones desde las Vanguardias Históricas. Es significativa la postura teórica de R. Roussel, detrás de las máquinas de hacer arte que propone en 1910, resultado de una contradicción entre el uso de aparatos modernos y una estética romántica desfasada. Marcel Duchamp, entre otros, expresaría años más tarde, la influencia que "Las visiones de África” ejerció en su trabajo.

\section{CONTEXTO DE UNA DISCUSIÓN MEDIATIZADA}

El fenómeno del arte en laboratorios es situado en la lógica conceptual del Arte tecnológico y/o la Estética científica y que ha sido objeto de estudio con profusión desde finales de los sesenta en occidente. Entre los resultados más difundidos, está la primera exposición de gráficos con computador, la Cybernetic Serendipity, Londres (1968). Hasta la exposición Les Immatériauxs en el Centro Pompidou (1985) y comisariado por J. Lyotard.

Experiencias que ulteriormente se reactivan bajo diversos nombres: Arte Medial, Mix Media, Arte mediático, o el de New Media Art y otros (Voropai, 2011). Es significativo que en la International Symposium on Electronic Art (ISEA, 2011) tampoco se llegó a consenso para su denominación: Computer art, Algorithmic art, Generative art, Inormation art, Evolutionary art, Process art, Systemic art, Cybernetic art, Kinetic art, Fractal art, etc. La profusión de términos evidencia la no consideración de investigaciones precedentes, y una ambigüedad al definir el arte desde los instrumentos. Tampoco es suficiente señalar que históricamente el término provendría de dos caminos separados: la tecnología informática y los mass media (Manovich, 2005).

Algunas posturas tienen antecedentes en la pretérita discusión entre el formalismo estético y la racionalidad conceptual, de allí la estética numérica, en la que su objetivo principal es alcanzar una convergencia entre las ciencias naturales y las del espíritu (Marchán, 1972). Ese formalismo de algunos autores los lleva a promover sin contrapeso analítico las relaciones arte-ciencias, descrita en proyectos como "El arte como criterio de excelencia. Modelo ARS" (Moraza y Cuesta, 2010: 6). Pues el desarrollo tecnológico tiene ramificaciones múltiples y ambiguas, y los artefactos y sus sistemas pueden ser utilizados para reproducir el orden social o para subvertirlo. "La tecnología puede ser y es configurada de un modo tal que reproduce el dominio de pocos sobre muchos" (Feenberg, 2005: 111). Ideológicamente, la cultura no está al margen del acontecer social, político o del poder del mercado (Feyerabend, 1986). Del mismo modo que la tecnología, para Benjamin, no sería un mero hecho científico, sino también histórico (2009: 78). 
La articulación que se busca entre los nuevos dispositivos digitales, los mass media y las ciencias, parece hacer referencia más a la forma que al contenido, en el sentido de diseño estético, disegno, o de la estética cientifica y del uso de la lógica estructural del lenguaje (Lippard, 2004). Contradiciendo la esencia del arte-ciencia que oscila en una constante búsqueda de violación de límites de la realidad, ya sea desde la ficción o la abstracción (Del Río, 2003). Concurrirían conceptos prejuiciados históricamente por los sistemas y estructuras establecidas, que los presentan como contrapuestos. Más bien existiría una relación y confluencias que permiten alcanzar comprensión de los modelos ideológicos personales (BensaudeVincent, 1991), y en las que el conocimiento es considerado concretamente político (Flusser, 2007).

\section{Referentes del Laboratorio Constructivista}

El aporte cardinal y pionero de las experiencias del ConstructivismoProductivista, fue sustentar una sólida y abundante reflexión artística con una praxis congruente con el contexto cultural-político-tecnológico. Esas primeras experiencias se plasmaron, por ejemplo, en construcciones tridimensionales que incorporan el espacio real dentro de sí misma. Motivados por unir lo formal y lo utilitario, la Unión de la Juventud -Soyuz- de San Petersburgo realiza una exposición en 1910. En esa búsqueda en 1919 sería fundada la sociedad de Artistas Jóvenes, OBMOKhU y en el año 1920 se forman los estatales Talleres de Enseñanza Superior de las Artes y las Técnicas, VKHUTEMAS, lo que sería la versión oriental de la Bauhaus de Weimar. En ellos se incuba la idea de arte como un conjunto de productos compuesto por diversos elementos y materiales organizados por los productores, es radicalmente opuesto al concepto de obra acomodada por elementos filosóficos, emocionales o de inspiración, aspectos vislumbrados por S. Straknov en la conferencia "La tecnología y la belleza de la vida" de 1905 (Lodder, 1988: 77). Para algunos investigadores partidarios de una revisión renovada, el influjo del arte ruso-soviético de 1910 a 1930 ha oscilado históricamente entre la fetichización de su formalismo y el arrebato o infamia de su idealismo, ahogando su carácter de acontecimiento poderoso y obviando su fuerte resonancia en la exploración política del arte y de transformación social (Expósito, 2010).

La actividades del Proletkult en Moscú a comienzos de la década de 1920, con sus proyectos de quioscos de Agit-prop produjeron agenciamientos mediáticos que eran el resultado de una concatenación de emisiones de radio, pantallas cinematográficas y prensa de propaganda, conectando así diferentes medios de comunicación con el fin de poner al servicio de la revolución nuevas invenciones que habían de ser funcionales al máximo. Los Constructivistas apostaban por un paso más allá en la política de adaptar el aparato de producción: las masas 
mismas habrían de convertirse en "autores como productores" (Raunig, 2009). Para Osborne una definición de Laboratorio Constructivista sería: “...laboratorio experimental formal en el horizonte del uso social" [...] "la construcción es un proceso racional e instrumental con condiciones sociales, materiales y tecnológicas históricamente específicas" (Osborne, 2010:173-175). Dentro de los distintos grupos de Constructivistas rusos, están los Constructivistas del Laboratorio, quienes abogan por una producción de objetos no utilitarios y que durante un período (1913-1920) establecieron los rasgos esenciales de la faktura, concepto relevante pues significaba además "...la incorporación a la obra de los medios técnicos de construcción y su vinculación con el estado de desarrollo de los medios de producción en la sociedad en general" (Buchloh, 2004:120-123). Un ejemplo de práctica artística productivista, es el cine-tren, (Alexander Medvedkin, cineasta responsable de la creación del proyecto en 1932, con el que recorrió el país) consistente en un estudio y laboratorio cinematográfico móvil. El tren se desplazaba a diferentes enclaves de producción para filmar en el lugar las prácticas de los trabajadores con el objetivo de revelar y editar el material de inmediato, para ser discutido directamente con sus protagonistas. Benjamin describiría tales prácticas como aquellas que intentaban cambiar el aparato de producción en lugar de limitarse a mostrar la tendencia política correcta (citado en Steyerl, 2009). Asimismo el Laboratorio Experimental de Construcciones Cinéticas del Proletkult de Moscú, buscaba probar experimentalmente todas las formas posibles de ensambladura social en el marco de talleres de formación: la conferencia, el banquete, el tribunal, la asamblea, la reunión, el auditorio, los acontecimientos y las competiciones deportivas, las veladas en el club, los vestíbulos, los comedores públicos, las celebraciones de masas, las procesiones, el carnaval, los funerales, los desfiles, las manifestaciones, las asambleas, el trabajo en la fábrica, las campañas electorales, etc. (Raunig, 2009). El Grupo de Constructivistas en Acción del Instituto de Cultura Artística, INKhUK (1921), exploran el planteamiento de cuestiones relacionadas con la ciencia y el arte en todos sus aspectos: desde la teoría de las artes, la teoría de la interacción y del arte como un todo (Lodder, 1988). El trabajo de Laboratorio, “...consistía en una experimentación abstracta, emprendida no por sí misma ni como respuesta a una exigencia utilitaria específica, sino con vistas a una aplicación en última instancia utilitaria" (Lodder, 1988: 225). Para Osborne (2010) la idea de "trabajo de Laboratorio" se refería a ejercicios formales llevados a cabo no por sí mismos sino como investigación para futuros usos instrumentales.

Los últimos estudios sobre el Constructivismo-Productivista apuntan a una necesaria revisión, ya que la distorsión histórica ha imposibilitado constatar la identidad política real de los Constructivistas. Impidiendo comprender uno de los conflictos más recónditos de la modernidad: "la dialéctica histórica entre 
la autonomía individual y la representación de la colectividad por medio de convenciones visuales" (Buchloh, 2004:145). En la actualidad algunos colectivos, están desarrollando nuevas prácticas artísticas que funden lo estético, el arte y el activismo, atendiendo a la historia de las rupturas que se oponen a las convenciones de la producción artística, e impulsa el desarrollo de un "Laboratorio vivo", emancipatorio y experimental (Vilensky, 2009). Asumiendo el riesgo, en estas nuevas estrategias políticamente emancipadoras y de inclusiones tecnológicas, con ser un instrumento eficaz de propaganda totalitaria y también en su adaptación a las necesidades de la industria cultural (Buchloh, 2004). El constructivismo respondía a la nueva época industrial al rechazar la actividad especulativa del arte y adherirse a una "producción intelectual y material". "Los artistas y teóricos culturales, compartían el convencimiento de que al modificar las condiciones de producción y recepción artísticas heredadas de la burguesía y sus instituciones, estaban participando en la transformación definitiva de la vanguardia moderna" (Buchloh, 2004: 117). En definitiva, las experiencias del Laboratorio Constructivista pusieron un énfasis radical entre la congruencia material del signo y la práctica significativa, curiosamente ese énfasis de relación trataba de desafiar la posibilidad de un nivel secundario de significado o interpretación.

Demarcando al Media Lab y espacios afines

$\mathrm{Al}$ revisar antecedentes del denonominado Media Lab en principio aparece identificado entre las actividades del New Media Art y la experimentación con tecnología digital. También es un hecho divulgado que el término Media Lab, que es un acrónimo proveniente del inglés Media Laboratory y cuya traducción original sería Laboratorios de medios de comunicación, se ha transformado en genérico de los espacios que trabajan con objetivos similares como son los: Laboratorios arte-ciencia (Lab Scienceart); Laboratorios vivientes (Living Lab); Laboratorios ciudadanos (City Lab); Laboratorios de fabricación (Maker Lab, Fab Lab); Laboratorios de comida (Food Lab); Laboratorios de activismo (Hacker Labs, o Hacker Spaces); Laboratorios temporales de medios (Temporary Media Labs) y la variación virtual que propone B. Latour (2003) los World Wide Lab.

También se asume, a fuerza de insistencia comunicacional (desde Brand, 1989 a Rowan, 2012), que el término se acuña y establece en el M.I.T. de Boston (USA) en 1985, en el grupo de Grupo de Arquitectura y Máquinas. Con el objetivo original de crear un laboratorio de producción de objetos para la industria informática y de telecomunicaciones. Sin embargo, habría que considerar otros hechos que modelan su génesis, y que son decisivas para comprender con mayor propiedad el fenómeno. Un aspecto que caracteriza a esta génesis es el afán ansioso de posicionamiento y figuración al publicitar el término Media Lab como nacido 
exclusivamente en un solo lugar y personalizado. Por ejemplo, Román Gubern (1989) investigador becado en el M.I.T., constata que el único centro de esa universidad desvinculada de la industria y la tecnología militar, es el Centro de Estudios Visuales Avanzados (CAVS). Desde el año 1967, Gyorgy Kepes (fundador de la Escuela Bauhaus de Weimar, en 1919), junto a otros profesores de la escuela de Arquitectura del M.I.T., concibe un programa de becas y el uso de los nuevos recursos tecnológicos en el arte con la interacción de artistas, científicos, ingenieros, y la industria, el entorno urbano y los medios de comunicación. Ante el beneficio publicitario que les reporta el CAVS conduce, de algún modo, a la propuesta de un nuevo centro de similares características.

Otro antecedente a considerar, es cuando el editor Jean-Jacques ServanSchreiber publica el libro "El desafio mundial" (1980) a propósito del repunte tecnológico y de la informatización de Japón. Tesis según la cual los trágicos desequilibrios que caracterizan el mundo serán superados por la fórmula: informatización universal, los petrodólares y la tecnología occidental (Beaumont, 1980). Diagnóstico que impulsa al Gobierno de F. Mitterrand a crear el instituto de informatización de Francia. Con el nombre de Centro Mundial de Informática y Recursos Humanos, bajo la presidencia de JJ.Servan-Schreiber y la dirección de N. Negroponte y S. Papert (Hernández, 1983). Finalmente, por problemas de fondos y a raíz de un informe del Tribunal de Cuentas, se cierra el proyecto en 1985. En esta experiencia surgen una serie de hechos que van desde el acuñamiento de nombres similares, la prioridad discursiva del uso de tecnologías para un futuro argumentado como promisorio, hasta la negociación con los gobiernos y empresas, cuya finalidad es vender proyectos y prototipos.

Entonces, N. Negroponte regresa al M.I.T. con la idea de continuar con un laboratorio que utilice herramientas digitales para el diseño de productos de consumo. Encargando la redacción de un libro con fines publicitarios (Brand, 1989) en el cual se promete un ambicioso futuro informático, interactivo, holográfico, de alta definición, etcétera (Ibídem). La ligadura de este Media Lab con las artes visuales resulta ser más bien superficial y una manifiesta operación cosmética. "Negroponte es originalmente arquitecto y tiene el cuidado de un artista en la calidad de toda presentación en la que participa" (Brand, 1989: 90).

\section{EL CONSTRUCTO DIFUNDIDO}

En consecuencia, existen una serie de iniciativas, hacia finales de los años sesenta en Occidente, que apuntan a producir y difundir trabajos artísticos y de diseño utilizando ordenadores y otras herramientas tecnológicas y de los medios

para comunicación de masas. Actualmente cientos de ellos se autoproclaman 
agentes de innovación, diseño y construcción que producen resultados concretos, dicen promover el intercambio interdisciplinar en un entorno de creación, e investigación. Vinculadas al ciberespacio término de la novela Neuromante de W. Gibson, junto a la expresión medios masivos de comunicación proveniente de un ensayo sobre el Pop art de L. Alloway, Las artes y los mass media.

Asimismo el término Living Labs es asociado a Williams J. Mitchell del Media Lab del M.I.T., quien hacia fines de los noventa, buscó un método para involucrar a los habitantes en la planificación urbana y el diseño de ciudades utilizando herramientas digitales (Pinto y Pedruzzi, 2013). Principalmente promovidos a través de la Red Europea de Living Lab Abiertos (ENoLL), espacios posibles de asociar a aspectos mediáticos teóricos como el de la sociedad del conocimiento, la industria cultural y la producción de prototipos, o también con el activismo, o la ecología. Poseen una estructura que aspira a explorar la cooperación y la interdisciplinariedad, aunque la complejidad cooperativa e interdisciplinar presenta enormes diferencias de grado entre un espacio y otro. Son disímiles los fines que persiguen, y la autodefinición no legitima la praxis sobre la experimentación artística cooperativa e interdisciplinar. Sería más acertado hablar de "grados de participación ciudadana" que se producirían en estos espacios (Arnstein, 1969).

La tecnología actual, según F. Jameson, es "bipnótica y fascinante"(1996: 57), pero, no por su atracción en sí misma, sino porque podría ofrecer en, su representación, un esquema privilegiado para comprender la red de poder y control, en la nueva red global. En la que recursos como la apropiación, la moda tecnológica y la mediática se confunden, de resultado provocador o lúdico que absorbe lo emancipador, diluyéndose en el "Entertainment" (Marchán, 2006), donde se publicita que los mass media amplían nuestros sentidos (MacLuhan y Powers, 2011), pero al parecer están más próximos a la suplantación, el asalto visual, el adormecimiento y el golpe de efecto en boga en muchos objetos de arte actuales. Por tanto, la investigación tecnocientífica, el diseńo industrial, y el desarrollo tecnológico para la innovación de productos o al arte, no son parámetros suficientes para entender la inserción social de un modo nuevo de producción con finalidad artístico-cultural. Así ocurre cuando atendemos a la tesis de Huhtamo (2010) quien precisa que la interacción con la vanguardia en publicidad y propaganda política, en el uso tecnológico y los mass media, ha sido anterior a la finalidad artística. Coincidente con la tesis propuesta por El Lissitzky en el artículo de 1921 "El artista y la producción", cuando señala que las prácticas del fotomontaje se iniciaron en la publicidad comercial estadounidense. Esto implica que la incorporación de nuevas tecnologías no es prioritaria en los procesos de autonomía colectiva. Por eso el punto débil de los actuales Laboratorios tecnológicos es la indiferencia ante la interacción necesaria, entre objeto de arte, proceso de producción y contexto cultural. Situados en el trasfondo de la pretérita paradoja del arte: Verdad versus Apariencia (Álvarez, 2007). 


\section{LA CUESTIÓN DEL INTERACCIONISMO EN LA PRODUCCIÓN DE ARTE}

Los procesos de arte serían el resultado de interacciones y acciones colectivas de los distintos "mundos del arte" certificados por las investigaciones de H. Becker (2008). Los procesos colaborativos y multidisciplinares, constituirían una alternativa al modelo profesional de propiedad, que utilizan patrones de colaboración en un clima de transversalidad (Buchmannn, 2009; Bain, 2007). Herramienta comunitaria que restablecería el equilibrio ecológico entre la cultura y el comercio y diferentes a las de cooperación en la fábrica y en su economía (Rifkin, 2000; Lazzarato, 2006). Contrarrestando, la mercantilización del conocimiento simbólico de los bienes públicos (Yúdice, 2002). Aunque entendemos que la intencionalidad colectiva de los seres humanos es compleja y las investigaciones sobre la génesis de la cooperación social humana, ya sea desde la psicología del desarrollo cognitivo, social, la neurobiología, la antropología cultural, la economía experimental o la sociología del arte, no son concluyentes (Tomasello, 2010).

¿Cómo evaluar el nivel de participación que acontece en los nuevos laboratorios?, o es que son incorporados comunidades marginadas por las mismas razones populistas que esgrime la institución burocrática (Yúdice, 2002) o sería más acertado hablar de "grados de participación ciudadana" que se producirían en estos espacios (Arnstein, 1969).

Un antecedente al trabajo cooperativo artístico inserto en la comunidad, son los conceptos de "Teamwork" y "Autoorganización" (von Bismarck, 2009), relacionados, aunque en la práctica sus raíces y contextos sociales son muy diferentes. Términos utilizados para designar orientaciones opuestas en las formas de trabajar colectivamente en el dominio del arte, exteriorizando una transición del constructo de la identidad del yo y el colectivo en el trabajo artístico (ArrazolaOńate, 2012). Fenómeno que permite comprender la producción del arte actual (Green, 2002), identificado por una búsqueda de diversos métodos de producción (Marín, 2009). Para estos nuevos espacios, la referencia más próxima sería el Floating Lab Collective's Re Museum, en Washignton DC, 2012, grupo que trabaja anónimamente y en el cual determinadas comunidades definen su concepto de arte y aportan sus producciones. En el lado opuesto, el proyecto de arte actual presentado en Art Labs de San Diego, EUA, 2013, el cual es una feria de arte profesional con una curaduría de representantes de centros de arte, universidades y editoriales asociadas a diseñadores de productos.

El concepto de trabajo colectivo de Floating Lab Collective's Re Museum es lo que para J. Rancière (2003) apunta al conocimiento no privativo de algunos, desmitificando las creencias de la autonomía del arte y la del genio. Son planteadas como actividades artísticas, que no difieren sustancialmente de otras clases de 
trabajo (Becker, 2008). Danto (2002) lo precisa en la "Teoría institucional del arte", donde las teorías artísticas y el contexto o dispositivo, hacen posible el mundo del arte. Emparentada con el mundo del arte de G. Dickie (1975), para quien la categoría de producto de arte se lo confieren las personas que representan la institución artística. Situación que la asociamos a los productivismos de 1910 en Europa oriental cuando las producciones experimentales sientan las bases de: una imbricación entre una estética, un proceso de producción y las relaciones sociales (Lodder, 1988). Grupos Experimentales de Construcciones y Laboratorios donde se trató de activar los nuevos medios de comunicación en espacios colectivos experimentales para organizar una producción artística de intercambio transversal.

\section{CONCLUSIONES}

Las denotaciones y connotaciones en un marco ideológico-histórico occidental han determinado un vocablo Laboratorio más bien comunicacional, que ha impedido una definición sólida. Bastamente se ha difundido sobre sus aspectos ideales y abstractos, como el uso de las últimas tecnologías, la aspiración de colaboración multidisciplinar y pretensiones de participación desjerarquizada. Aunque poco es el análisis sobre su estructura social cultural-político y sus redes ideológicas, también ha contribuido el silencio observado frente al cambio de paradigma propuesto en el programa constructivista-productivista, que recién casi ochenta años después es retomado por algunas investigaciones académicas, ya sea por su extraordinaria productividad de la vanguardia moderna original, por el número de participantes o la variedad de su producción. En cambio, el alcance de los Laboratorios propuestos desde los años ochenta, más bien evidencian connotaciones que están ligadas a la empresa y la venta de prototipos. Con escasa presencia en proyectos artísticos, el término arte se presenta allí más como efecto mediático que otra cosa. Lo más probable es que por allí se reste capacidad de resistencia y dimensión de análisis, y aumente la distracción y la capacidad productiva mercantil.

La interacción Laboratorios de acción participativa y experimental requiere de una fundamentación crítica genealógica e ideológica. Específicamente, la producción artística plástica requiere de reales espacios participativos y experimentales. Opuesto a la suplantación, el asalto visual y el adormecimiento o modo de protección ante la manipulación de la experiencia. Además la producción artística requiere de análisis e investigadores abiertos, autocríticos y reflexivos con teorías, conceptos y términos instalados en espacios de interacción. Del mismo modo construir mecanismos de defensa ante el proceso mercantil del sistema del arte que copa el espacio cultural. 


\section{REFERENCIAS}

ATLAS, Walter Benjamin. "El autor como productor". Obras, Libro II. Volumen 2. Madrid: Abada, (2009): 297-315.

Álvarez, Luis. "La aporía del arte: "Hipertrofia" del entendimiento y "represión de la sensibilidad", Fedro, Revista de estética y teoría del arte 5 (2007): 50-75.

Arnstein, Sherry. "A Ladder of Citizen Participation", Journal of the American planning Associaton, 35/4 (1969): 216-234.

Arrazola- Ońate, Txaro. "Creación colectiva. Teorías sobre la noción de autoría, modelos colaborativos de creación e implicaciones para la práctica y la educación”. Tesis doctoral. España: Universidad del País Vasco, (2012).

Bain, Ken. Lo que hacen los mejores profesores universitarios. Valencia: Universitat de València, 2007.

Beaumont, José. Jean-Jacques Servan-Schreiber presentó en Madrid su último "desafio". Diario EL PAÍS. 27 de noviembre 1980.

Becker, Howard. Mundos del arte. Sociología del trabajo artístico. Buenos Aires: Quilmes, 2008.

Benjamin, Walter. Walter Benjamin: Obras libro II. Volumen 1. Madrid: Abada Editores, 2008.

Benjamin, Walter. "El autor como productor", Tentativas sobre Brecht. Iluminaciones III, Madrid: Taurus, 1990. $3^{a}$ edición. (1990):107-120.

Bensaude-Vincent, Bernadette. "Lavoisier: Una revolución científica". En Serres Michel (ed.), Historia de las ciencias. Madrid: Cátedra, (1991): 411-435.

Borgdorff, Henk. "El debate sobre la investigación en las artes", Cairon: Revista de ciencias de la danza, 13 (2006): 25-46.

Brand, Stewart. El Laboratorio de Medios. Inventando el futuro en el M.I.T. Madrid: Fundesco, 1989. 
Buchloh, Benjamin. Formalismo e historicidad. Modelos y métodos en el arte del siglo XX. Madrid: Akal, 2004.

Buchmann, Sabeth. "Art \& Language". Butin Hubertus (ed.), Diccionario de conceptos de arte contemporáneo. Madrid: Abada, (2009): 21-24.

Danto, Arthur. La transfiguración del lugar común. Una filosofía del arte. Barcelona: Paidós, 2002.

Del Río, Víctor. "El espacio ideológico del arte y la ciencia”, Revista Lápiz, Revista Internacional de Arte, año XIX, 193, (2003): 28-39.

Dickie, George. El círculo del arte. Una teoría del arte. Barcelona: Paidós Estética, 2005.

Expósito, Marcelo. "Los nuevos productivismos", 2010. Disponible en: http://eipcp. net/transversal/0910/exposito/es. [Consultado: el 22 de marzo de 2013].

Feenberg, Andrew. "Teoría crítica de la tecnología”, Revista CTS, 5/ 2, (2005): 109-123.

Feyerabend, Paul. Tratado contra el método. Esquema de una teoría anarquista del conocimiento. Madrid: Tecnos, 1986.

Flusser, Vilém. Sobre arte, aparatos y funcionarios, 2007. Disponible en: http:// www.revista-artefacto.com.ar/pdf_notas/162.pdf.[Consultado: el 12 de abril de 2010].

Green, Charles. "La tercera mano: Colaboración y arte contemporáneo", Exit special issue, Teamwork, Madrid, 7 (2002): 96-125.

Gubern, Román. "Prólogo". El Laboratorio de Medios. Inventando el futuro en el M.I.T. Steward Brand. Madrid: Fundesco, (1989): 13-17.

Hernández, José. "La Revolución informática transformará a Colombia", 1983. Disponible en: http://www.disi.unal.edu.co/-gjhernandezp/introisc/hide/ RevolucionInformatica TransformaraColombia1983Semana.pdf.[Consultado: el 15 de diciembre de 2012]. 
Huhtamo, Erkki. "Una arqueología multimedia de altura", 2010. Disponible en: http://www.medialabprado.es/article/open_up.[Consultado: el 10 de febrero de 2010].

Jameson, Fredric. Teoría de la postmodernidad. Madrid: Trotta, 1996.

Latour, Bruno."El Laboratorio de la World Wide", 2003. Disponible en: http:// www.wired.com/wired/archive/11.06/research_spc.html.[Consultado: el 3 de abril de 2011].

Lazzarato, Maurizio. Por una política menor. Acontecimiento y politicas en las sociedades de control. Madrid: Traficantes de sueños, 2006.

Lippard, Lucy. Seis años: La desmaterialización del objeto artístico de 1966 a 1972. Madrid: Akal / arte contemporáneo, 2004.

Lodder, Christina. El constructivismo ruso. Madrid: Alianza, 1988.

MacLuhan, Marshall y Powers, Bruce R. La aldea global. Transformaciones en la vida y los medios de comunicación mundiales en el siglo XXI. Barcelona: Gedisa, 2011.

Manovich, Lev. El lenguaje de los nuevos medios de comunicación. La imagen en la era digital. Barcelona: Paidós, 2005.

Marchán, Simón (comp.), Reall virtual en la estética y la teoría de las artes. Barcelona: Paidós, 2006.

Marchán, Simón. "La "estética científica", de Max Bense". Estética de la información. Introducción a la estética teórico-informacional. Fundamentación y aplicación a la teoría del texto. Madrid: Alberto Corazón, (1972):7-17.

Marín, Teresa. "Estrategias de colectiva en el arte contemporáneo", 2009. Disponible en: http://cmap.javeriana.edu.co/servlet/SBReadResourceServ let?rid=1J2NZ94P4-1S827PX-1S9. [Consultado: el 9 de marzo de 2010].

Moraza, Juan y Cuesta, Salomé. El Arte como criterio de excelencia. Modelo ARS (Art: Research: Society). Madrid: Ministerio de Educación, 2010. 
Osborne, Peter. El arte más allá de la estética. Ensayos filosóficos sobre arte contemporáneo. Murcia: Ad Litteram Cendeac, 2010.

Pinto, Miriam y Pedruzzi, Letizia. "Profundizando la comprensión de los Living Labs de Brasil", Revista CTS 8/ 5, (2013): 231-247.

Rancière, Jacques. El maestro ignorante. Cinco lecciones sobre la emancipación intelectual. Barcelona: Laertes, 2003.

Raunig, Gerald. "Transformar el aparato de producción. La concepción de una intelectualidad antiuniversalista en la temprana Unión Soviética”, 2009. Disponible en: http://eipcp.net/transversal/0910/raunig/es. [Consultado: el 9 de julio de 2012].

Rifkin, Jeremy. La era del acceso. La revolución de la nueva economía. Barcelona: Paidós, 2000.

Sangüesa, Ramón. "La tecnocultura y su democratización: Ruido, límites y oportunidades de los Labs", Revista CTS, 8/ 5 (2013): 259-282.

Serra, Artur. "Citilabs: ¿Qué pueden ser los laboratorios ciudadanos?”, 2010. Disponible en: http://www.revistalafactoria.eu/articulo.php?id=523. [Consultado: el $22 \mathrm{de}$ agosto de 2012].

Steyerl, Hito. "La verdad desecha. Productivismo y factografía", 2009. Disponible en: htttp://eipcp.net/transversal/0910/steyerl/es. [Consultado: el $11 \mathrm{de}$ junio de 2012].

Tomasello, Michael. ¿Por qué cooperamos? Madrid: Katz Editores, 2010.

Vilensky, Dmitry. El «club activista» o sobre los conceptos de casa de cultura, centro social y museo", 2009. Disponible en: http://eipcp.net/transversal/0910/ vilensky/es. [Consultado: el 28 de septiembre de 2012].

Von Bismarck, Beatrice. "Teamwork y Autoorganización”. En Hubertus Butin (ed.), Diccionario de conceptos de arte contemporáneo. Madrid: Abada, (2009): 211-213. 
Voropai, Lioudmila. "Theoretical Discourse on "Art, Science and Technology Collaboration" and its Historical Development", 2011. Disponible en: http:// isea2011.sabanciuniv.edu/paper/theoretical-discourse-\%E2\%80\%9Cartscience-and-technology-collaboration $\% \mathrm{E} 2 \% 80 \% 9 \mathrm{D}$-and-its-historicaldevelopmen. [Consultado: el 11 de febrero de 2012].

Yúdice, George. El recurso de la cultura. Usos de la cultura en la era global. Barcelona: Gedisa, 2002. 